

\title{
Discrete-Time BAM Neural Networks With Variable Delays
}

\author{
${ }^{*}$ Xin-Ge Liu ${ }^{a}$, Mei-Lan Tang ${ }^{a}$, Ralph Martin ${ }^{b}$, Xin-Bi Liu ${ }^{c}$ \\ ${ }^{a}$ School of Mathematical Science and Computing Technology, Central South University \\ Changsha, Hunan 410083, China \\ ${ }^{b}$ School of Computer Science, Cardiff University, Cardiff, UK \\ ${ }^{c}$ School of Materials Science and Engineering, Central South University \\ Changsha, Hunan 410083, China
}

\begin{abstract}
This letter deals with the global exponential stability of discrete-time bidirectional associative memory (BAM) neural networks with variable delays. Using a Lyapunov functional, and linear matrix inequality techniques (LMI), we derive a new delay-dependent exponential stability criterion for BAM neural networks with variable delays. As this criterion has no extra constraints on the variable delay functions, it can be applied to quite general BAM neural networks with a broad range of time delay functions. It is also easy to use in practice. An example is provided to illustrate the theoretical development.

Keywords: BAM neural network; Discrete-time; Global exponential stability; Variable delay; Linear matrix inequality (LMI); Delay-dependent.
\end{abstract}

\section{Introduction}

The dynamics of neural networks have been extensively investigated. The BAM neural network model, an extension of the unidirectional autoassociator of Hopfield [1], was first introduced by Kosko $[2,3]$. A BAM neural network is composed of neurons arranged in two layers: the $X$ and $Y$-layers. The neurons in one layer are fully interconnected to the neurons in the other layer and have associated connection weights. Through iterations of forward and backward information flows between the two layers, the network performs a two-way associative search for stored bipolar vector pairs, and generalizes the single-layer autoassociative Hebbian correlation to a two-layer pattern matched heteroassociative circuit. BAM neural networks have potential applications in many fields such as artificial intelligence. Many papers have studied the stability of this type of neural networks model (see, for example, [4] - [12]). The stability criteria for continuous-time BAM neural network models obtained in [13]-[17] are explicit and easily verified in practice. However, these stability criteria only take into account the absolute values of the entries in the connection matrices, neglecting their signs, and thus the differences between neuronal excitatory and inhibitory effects are ignored.

Recently, many papers have focused on the dynamics of continuous-time BAM neural networks. Although several papers (e.g. [16],[18]-[23]) have studied the dynamics of certain discrete-time neural networks, few papers have studied the stability of discrete-time BAM neural networks. Results concerning the exponential stability of discrete-time BAM neural networks with variable delays are fewer. However, in numerical simulations and practical implementations continuoustime neural networks, discretization is necessary. We note that in practice, the dynamics of discrete-time neural networks may be quite different from those of continuous-time ones. In particular, the stability criteria established for continuous-time BAM neural networks model are not necessarily applicable to discrete-time systems. Therefore, the dynamics of discrete-time neural networks are of both theoretical and practical importance.

${ }^{*}$ Corresponding Author. Tel.: +86-731-8830812. E-mail address: liuxgliuhua@163.com 
Recently, Wang and Zou [24] employed various fundamental mathematical techniques instead of the bifurcation method to investigate the existence, stability and number of stable periodic solutions for discrete-time BAM neural networks with constant delays. Applying Lyapunov functionals and Halanay-type inequalities, Mohamad [16] derived delay-independent sufficient conditions under which continuous-time BAM neural networks converge exponentially to the equilibria associated with temporally uniform external inputs to the networks. Discrete-time analogues of continuous-time BAM neural networks were formulated and their dynamical characteristics studied in [16]. However, the exponential convergence sufficiency conditions derived in [16] are only applicable to BAM neural networks with constant delays.

Using $M$-matrix methods, Liang and Cao [25] studied the exponential stability of continuoustime BAM with constant delays and its discrete analogue. Liang et al. [26] also studied the dynamics of discrete-time BAM neural networks with variable time delays $k(n)$ and $l(n)$ in the system model (see later for definitions), where $n$ represents discrete time. They obtained various delay-dependent and delay-independent stability criteria. However, their delay-dependent criteria are based on unreasonably severe constraints on the delay functions: $1<k(n+1)<1+k(n)$ and $1<l(n+1)<1+l(n)$. Most delay functions in practice do not satisfy such severe constraints.

The purpose of this paper is to further consider the global exponential stability of discretetime BAM neural networks. Our results avoid the constraints on delay functions in [26]. Using a new Lyapunov functional, and techniques involving linear matrix inequalities (LMIs), we are able to give a new delay-dependent stability criterion for BAM neural networks with variable delays. Since our result imposes few constraints on the system, it can be applied to more general BAM neural networks with a broad range of time delay functions. Moreover, our criterion is easy to check and apply in practice, and is thus of significance and interest both to application fields, and the design of neural networks.

The following notation is used: $\lambda_{\max }(Q)$ and $\lambda_{\min }(Q)$ denote the maximum and minimum eigenvalues of a symmetric matrix $Q . Q^{T}$ and $Q^{-1}$ denote its transpose and inverse. $Q>0$ (or $Q \geq 0$ ) means that $Q$ is a real, symmetric and positive definite (or semi-positive definite) matrix. $\|Q\|=\left(\lambda_{\max }\left(Q^{T} Q\right)\right)^{1 / 2}$. We write $N(-k, 0)$ for $\{-k,-k+1, \ldots, 1,0\}, N(1, m)$ for $\{1, \ldots, m\}$, and $N(k)$ for $\{k, k+1, \ldots\}$. We define $x=\left(x_{1}, x_{2}, \ldots, x_{m}\right)^{T} \in R^{m}$, and $\|x\|=\left(\sum_{i=1}^{m} x_{i}^{2}\right)^{1 / 2}$. We use the notation $k_{\min }=\min \{k(n)\}, k_{\max }=\max \{k(n)\}, l_{\min }=\min \{l(n)\}$, and $l_{\max }=\max \{l(n)\}$.

\section{System description}

Consider the following discrete-time BAM neural network with time-varying delays described by a set of non-linear difference equations

$$
\begin{aligned}
& x_{i}(n+1)=a_{i} x_{i}(n)+\sum_{j=1}^{m} c_{i j} f_{j}\left(y_{j}(n-k(n))\right)+I_{i} \\
& y_{j}(n+1)=b_{j} y_{j}(n)+\sum_{i=1}^{m} d_{j i} g_{i}\left(x_{i}(n-l(n))\right)+J_{j}
\end{aligned}
$$

where $i, j \in N(1, m) \cdot x_{i}(n)$ and $y_{j}(n)$ are the states of the $i^{\text {th }}$ neuron from the neural field $F_{X}$ and the $j^{\text {th }}$ neuron from the neural field $F_{Y}$ at time $n$, respectively, where $x(n)=\left(x_{1}(n), \ldots, x_{m}(n)\right)^{T}$ and $y(n)=\left(y_{1}(n), \ldots, y_{m}(n)\right)^{T} . a_{i}, b_{j} \in(0,1)$ describe the stability of internal neuron processes on the $X$-layer and the $Y$-layer, respectively. $f_{j}(\cdot)$ and $g_{i}(\cdot)$ denote the activation functions of the $j^{\text {th }}$ neuron from the neural field $F_{Y}$ and the $i^{\text {th }}$ neuron from the neural field $F_{X}$, respectively. The connection weights $c_{i j}$ and $d_{j i}$ are real numbers, which denote the strengths of connectivity between the $i^{\text {th }}$ neuron from the neural field $F_{X}$ and the $j^{\text {th }}$ neuron from the neural field $F_{Y} \cdot k(n)$ and $l(n)$ represent time delays with nonnegative integer values, bounded by $k_{\min } \leq k(n) \leq k_{\max }$ and $l_{\min } \leq l(n) \leq l_{\max } . I_{i}$ and $J_{j}$ denote the external constant inputs from outside the network acting on the $i^{\text {th }}$ neuron from the neural field $F_{X}$ and the $j^{\text {th }}$ neuron from the neural field $F_{Y}$, respectively. 
The initial conditions associated with the BAM neural network given in Eq. (1) are

$$
x_{i}(s)=\phi_{i 1}(s), \quad y_{j}(s)=\phi_{j 2}(s), \quad s \in N\left(-k^{*}, 0\right),
$$

where $k^{*}=\max \left\{k_{\max }, l_{\max }\right\}$.

Definition 1. The vector $\left(\begin{array}{c}x^{*} \\ y^{*}\end{array}\right)=\left(x_{1}^{*}, x_{2}^{*}, \ldots, x_{m}^{*}, y_{1}^{*}, y_{2}^{*}, \ldots, y_{m}^{*}\right)^{T}$ is said to be an equilibrium point of the BAM neural network given in Eq. (1) if it satisfies

$$
\begin{aligned}
& x_{i}^{*}=a_{i} x_{i}^{*}+\sum_{j=1}^{m} c_{i j} f_{j}\left(y_{j}^{*}\right)+I_{i}, \\
& y_{j}^{*}=b_{j} y_{j}^{*}+\sum_{i=1}^{m} d_{j i} g_{i}\left(x_{i}^{*}\right)+J_{j} .
\end{aligned}
$$

Definition 2. The BAM neural network given in Eq. (1) is said to be exponentially stable if for the equilibrium point $\left(\begin{array}{c}x^{*} \\ y^{*}\end{array}\right)$ there exist scalars $r>1$ and $\theta>1$ such that

$$
\left.\left\|x(n)-x^{*}\right\|^{2}+\left\|y(n)-y^{*}\right\|^{2} \leq \theta r^{-n}\left(\sup _{s \in N\left(-l_{\max }, 0\right)}\left\|x(s)-x^{*}\right\|^{2}+\sup _{s \in N\left(-k_{\max }, 0\right)} \| y(s)-y^{*}\right) \|^{2}\right) .
$$

Through this paper, we assume:

Assumption 1. The non-linear activation functions $f_{j}(\cdot), g_{i}(\cdot)$ are bounded and globally Lipschitz, i.e. they satisfy

$$
\begin{aligned}
\left|f_{j}(u)\right| & \leq S_{j 1}, & & \left|f_{j}(u)-f_{j}(v)\right| \leq M_{j}|u-v|, \\
\left|g_{i}(u)\right| & \leq S_{i 2}, & & \left|g_{i}(u)-g_{i}(v)\right| \leq L_{i}|u-v|,
\end{aligned}
$$

for $i, j \in N(1, m)$ and $u, v \in R$ where $S_{j 1}, S_{i 2}, M_{j}$, and $L_{i}$ are positive constants.

Assumption 1 ensures that the BAM neural network given in Eq. (1) has an equilibrium point

$$
\left(\begin{array}{c}
x^{*} \\
y^{*}
\end{array}\right) \text {. }
$$

Changing notation for simplicity, let $u_{i}(n)=x_{i}(n)-x_{i}^{*}, v_{j}(n)=y_{j}(n)-y_{j}^{*}$. We can rewrite Eq. (1) as

$$
\begin{aligned}
& u(n+1)=A u(n)+C \varphi(v(n-k(n))), \\
& v(n+1)=B v(n)+D \psi(u(n-l(n))),
\end{aligned}
$$

where $u(n)=\left(u_{1}(n), \ldots, u_{m}(n)\right)^{T}, v(n)=\left(v_{1}(n), \ldots, v_{m}(n)\right)^{T}, C=\left(c_{i j}\right)_{m \times m}, \quad D=\left(d_{i j}\right)_{m \times m}$, $A=\operatorname{diag}\left(a_{1}, \ldots, a_{m}\right), B=\operatorname{diag}\left(b_{1}, \ldots, b_{m}\right), \varphi(v(n))=\left(\varphi_{1}\left(v_{1}(n)\right), \ldots, \varphi_{m}\left(v_{m}(n)\right)\right)^{T}, \varphi_{j}\left(v_{j}(n)\right)=$ $f_{j}\left(v_{j}(n)+y_{j}^{*}\right)-f_{j}\left(y_{j}^{*}\right), \psi(u(n))=\left(\psi_{1}\left(u_{1}(n)\right), \ldots, \psi_{m}\left(u_{m}(n)\right)\right)^{T}$, and $\psi_{i}\left(u_{i}(n)\right)=g_{i}\left(u_{i}(n)+x_{i}^{*}\right)-$ $g_{i}\left(x_{i}^{*}\right)$.

For $s \in R$, Assumption 1 implies that

$$
\begin{aligned}
\left|\varphi_{j}(s)\right| & \leq 2 S_{j 1}, & & \left|\varphi_{j}(s)\right| \leq M_{j}|s| ; \\
\left|\psi_{i}(s)\right| & \leq 2 S_{i 2}, & & \left|\psi_{i}(s)\right| \leq L_{i}|s| .
\end{aligned}
$$

Therefore, global exponential stability of the BAM neural network given in Eq. (1) is equivalent to global exponential stability at the origin of the BAM neural network given in Eq. (4).

The problem to be addressed in this paper is to develop a new delay-dependent condition such that the discrete-time BAM neural network given in Eq. (1) is globally exponentially stable. More specifically, a new delay-dependent stability criterion for BAM neural networks with variable delays will be given. We avoid the severe constraints imposed on the delay functions necessary in [26] and extend certain results in [16] to the case with time-varying delays. 


\section{Main result}

This section considers the global exponential stability of the discrete-time delayed BAM neural network given in Eq. (1). We state the result as a theorem:

Theorem 1. Under Assumption 1, the origin of Eq. (4) is globally exponentially stable if a number $r>1$, positive definite matrices $P, Q$, and positive diagonal matrices $\Sigma_{1}, \Sigma_{2}$ exist such that

$$
\begin{aligned}
& {\left[\begin{array}{cc}
r A P A-P+F & r A P C \\
r C^{T} P A & r C^{T} P C-r^{-k_{\max } \Sigma_{1}}
\end{array}\right]<0,} \\
& {\left[\begin{array}{cc}
r B Q B-Q+G & r B Q D \\
r D^{T} Q B & r D^{T} Q D-r^{-l_{\max } \Sigma_{2}}
\end{array}\right]<0,}
\end{aligned}
$$

and furthermore,

$$
\|u(n)\|^{2}+\|v(n)\|^{2} \leq \theta r^{-n}\left(\sup _{s \in N\left(-l_{\max }, 0\right)}\|u(s)\|^{2}+\sup _{s \in N\left(-k_{\max }, 0\right)}\|v(s)\|^{2}\right)
$$

where $\theta=d / \min \left\{\lambda_{\min }(P), \lambda_{\min }(Q)\right\}, d=\max \left\{\lambda_{\max }(P)+\left(l_{\max }-l_{\min }+1\right) \delta_{1} \lambda_{\max }\left(\Sigma_{2}\right)\|L\|^{2}, \lambda_{\max }(Q)+\right.$ $\left.\left(k_{\max }-k_{\min }+1\right) \delta_{2} \lambda_{\max }\left(\Sigma_{1}\right)\|M\|^{2}\right\}, \delta_{1}=\left(1-r^{-l_{\max }}\right) /(r-1), \delta_{2}=\left(1-r^{-k_{\max }}\right) /(r-1)$, $F=\left(l_{\max }-l_{\min }+1\right) L \Sigma_{2} L, G=\left(k_{\max }-k_{\min }+1\right) M \Sigma_{1} M, L=\operatorname{diag}\left(L_{1}, \ldots, L_{m}\right)$, and $M=$ $\operatorname{diag}\left(M_{1}, \ldots, M_{m}\right)$.

The proof of Theorem 1 is given in the Appendix.

Theorem 1 in this paper is simpler than the criterion arising from Theorem 1 in [26] which contains four separate LMIs which must be satisfied.

\section{Discretization}

In this section, we propose a method for formulating a discrete-time analogue of continuous-time BAM neural networks, and analyze the dynamical characteristics of continuous-time BAM neural networks using Theorem 1 above.

A class of continuous-time BAM neural networks with time delays is described by the following set of differential equations:

$$
\begin{aligned}
& \dot{x}_{i}(t)=-\tilde{a}_{i} x_{i}(t)+\sum_{j=1}^{m} \tilde{c}_{i j} f_{j}\left(y_{j}(t-\tau(t))\right)+\tilde{I}_{i}, \\
& \dot{y}_{j}(t)=-\tilde{b}_{j} y_{j}(t)+\sum_{i=1}^{m} \tilde{d}_{j i} g_{i}\left(x_{i}(t-\sigma(t))\right)+\tilde{J}_{j} .
\end{aligned}
$$

where $x_{i}(t)$ and $y_{j}(t)$ are the states of the $i^{\text {th }}$ neuron from the neural field $F_{X}$ and the $j^{\text {th }}$ neuron from the neural field $F_{Y}$ at time $t$, respectively. We assume the activation functions $f_{j}(\cdot)$, $g_{i}(\cdot)$ satisfy Assumption $1 . \tilde{I}_{i}$ and $\tilde{J}_{j}$ denote external contant inputs to the $i^{\text {th }}$ neuron from the neural field $F_{X}$ and the $j^{\text {th }}$ neuron from the neural field $F_{Y}$, respectively. $\tilde{a}_{i}>0$ and $\tilde{b}_{j}>0$ denote the rates with which these neurons reset their potentials to the resting state in isolation when disconnected from the network and external inputs, respectively. $\tilde{c}_{i j}$ and $\tilde{d}_{j i}$ are constants denoting connection strengths. Time delays $\tau(t)$ and $\sigma(t)$ are nonnegative functions, satisfying $0 \leq \tau(t)<\tau$ and $0 \leq \sigma(t)<\sigma ; \tau$ and $\sigma$ are positive constants.

We reformulate the network in Eq. (9) using the following approximation:

$$
\begin{aligned}
& \dot{x}_{i}(t)=-\tilde{a}_{i} x_{i}(t)+\sum_{j=1}^{m} \tilde{c}_{i j} f_{j}\left(y_{j}([t / h] h-[\tau(t) / h] h)\right)+\tilde{I}_{i}, \\
& \dot{y}_{j}(t)=-\tilde{b}_{j} y_{j}(t)+\sum_{i=1}^{m} \tilde{d}_{j i} g_{i}\left(x_{i}([t / h] h-[\sigma(t) / h] h)\right)+\tilde{J}_{j},
\end{aligned}
$$


where $i, j \in N(1, m) . h$ is a fixed positive real number denoting a uniform arbitrary step-size and $[r]$ denotes the integer part of the real number $r . t \in[n h,(n+1) h) ; n$ is a non-negative integer. For convenience, we use the notation $p_{i}(n)$ and $q_{j}(n)$ to stand for $x_{i}(n h)$ and $y_{j}(n h)$, respectively. Clearly, for $t \in[n h,(n+1) h)$, we have $[t / h]=n$. Let $k(n)=[\tau(t) / h], l(n)=[\sigma(t) / h], k_{\max }=[\tau / h]$, $l_{\max }=[\sigma / h], k^{*}=\max \left\{k_{\max }, l_{\max }\right\}$. With these preparations we may rewrite the BMA neural network in Eq. (10) as

$$
\begin{aligned}
& \dot{x}_{i}(t)=-\tilde{a}_{i} x_{i}(t)+\sum_{j=1}^{m} \tilde{c}_{i j} f_{j}\left(q_{j}(n-k(n))\right)+\tilde{I}_{i}, \\
& \dot{y}_{j}(t)=-\tilde{b}_{j} y_{j}(t)+\sum_{i=1}^{m} \tilde{d}_{j i} g_{i}\left(p_{i}(n-l(n))\right)+\tilde{J}_{j} .
\end{aligned}
$$

Integrating both sides of Eq. (11) over $[n h, t)$ where $n h \leq t<(n+1) h$ gives

$$
\begin{aligned}
& x_{i}(t)=e^{\tilde{a}_{i}(n h-t)} p_{i}(n)+\left(1-e^{\tilde{a}_{i}(n h-t)}\right)\left\{\sum_{j=1}^{m} \tilde{c}_{i j} f_{j}\left(q_{j}(n-k(n))\right)+\tilde{I}_{i}\right\} / \tilde{a}_{i}, \\
& y_{j}(t)=e^{\tilde{b}_{j}(n h-t)} q_{j}(n)+\left(1-e^{\tilde{b}_{j}(n h-t)}\right)\left\{\sum_{i=1}^{m} \tilde{d}_{j i} g_{i}\left(p_{i}(n-l(n))\right)+\tilde{J}_{j}\right\} / \tilde{b}_{j} .
\end{aligned}
$$

Let $t \rightarrow(n+1) h$ in Eq. (12), giving

$$
\begin{aligned}
& p_{i}(n+1)=e^{-\tilde{a}_{i} h} p_{i}(n)+\left(1-e^{-\tilde{a}_{i} h}\right)\left\{\sum_{j=1}^{m} \tilde{c}_{i j} f_{j}\left(q_{j}(n-k(n))\right)+\tilde{I}_{i}\right\} / \tilde{a}_{i}, \\
& q_{j}(n+1)=e^{-\tilde{b}_{j} h} q_{j}(n)+\left(1-e^{-\tilde{b}_{j} h}\right)\left\{\sum_{i=1}^{m} \tilde{d}_{j i} g_{i}\left(p_{i}(n-l(n))\right)+\tilde{J}_{j}\right\} / \tilde{b}_{j} .
\end{aligned}
$$

One can show that the discrete-time analogue Eq. (13) converges to the continuous-time BAM neural networks Eq. (9) when $h \rightarrow 0^{+}$. Let

$$
\begin{aligned}
& a_{i}=e^{-\tilde{a}_{i} h}, \quad c_{i j}=\frac{1-e^{-\tilde{a}_{i} h}}{\tilde{a}_{i}} \tilde{c}_{i j}, \quad I_{i}=\frac{1-e^{-\tilde{a}_{i} h}}{\tilde{a}_{i}} \tilde{I}_{i}, \\
& b_{j}=e^{-\tilde{b}_{j} h}, \quad d_{j i}=\frac{1-e^{-\tilde{b}_{j} h}}{\tilde{b}_{j}} \tilde{d}_{j i}, \quad J_{j}=\frac{1-e^{-\tilde{b}_{j} h}}{\tilde{b}_{j}} \tilde{J}_{j} .
\end{aligned}
$$

When the step-size $h$ is small enough, the question of stability of Eq. (9) can be reduced to the stability of the following discrete-time BAM neural network

$$
\begin{aligned}
& p_{i}(n+1)=a_{i} p_{i}(n)+\sum_{j=1}^{m} c_{i j} f_{j}\left(q_{j}(n-k(n))\right)+I_{i}, \\
& q_{j}(n+1)=b_{j} q_{j}(n)+\sum_{i=1}^{m} d_{j i} g_{i}\left(p_{i}(n-l(n))\right)+J_{j} .
\end{aligned}
$$

Therefore, we can use Theorem 1 above to analyze the the global exponential stability of continuoustime BAM neural networks with variable delays. In fact, if the discrete-time analogue of a continuous-time BAM neural network is globally exponentially stable with a small enough uniform arbitrary step-size $h$, then the continuous-time BAM neural network is globally exponentially stable. But we should note that the discrete-time analogue in this paper may not preserve the convergence characteristics of the associated continuous-time BAM neural networks without restriction on the size of the uniform discretization step. 
Remark 1. Mohamad [16] and Liang and Cao [25] studied the globally exponential stability of time-delayed BAM neural networks in the case where the time delays $\tau(t)$ and $\sigma(t)$ are constants. Mohamad [16] proved that if $\tilde{a}_{i}>L_{i} \sum_{j=1}^{m}\left|\tilde{d}_{j i}\right|$ and $\tilde{b}_{j}>M_{j} \sum_{i=1}^{m}\left|\tilde{c}_{i j}\right|$, the continuous-time BAM neural network in Eq. (9) and its discrete analogue are globally exponentially stable. Liang and Cao [25] obtained other global exponential stability criteria for the continuous-time BAM neural network in Eq. (9) and its discrete analogue. However, all stability criteria given in [16, 25] are delay-independent, and furthermore depend on the absolute values of the connection weights $\tilde{c}_{i j}$ and $\tilde{d}_{j i}$, i.e. $\left|\tilde{c}_{i j}\right|$ and $\left|\tilde{d}_{j i}\right|$, instead of $\tilde{c}_{i j}$ and $\tilde{d}_{j i}$. Since these stability criteria neglect the signs of the entries in the connection matrices, differences between neuronal excitatory and inhibitory effects are ignored, as is also the case in [27]-[30]. Furthermore, the delays in [16, 25] are both time-invariant. In contrast, the stability criterion in our paper takes into account the signs of the entries in the connection matrices, and furthermore is applicable to discrete-time BAM neural networks with time-varying delays.

Remark 2. Using a similar discretizing method to that in [18], Liang et al [26] earlier discretized the continuous-time BAM neural network in Eq. (9) into the discrete-time BAM neural network in Eq. (1) with delay functions $k(n)$ and $l(n)$. They obtained various delay-dependent and delayindependent stability criteria for the discrete-time BAM neural network in Eq. (1). However, their delay-dependent criteria are based on unreasonably severe constraints on the delay functions, requiring $1<k(n+1)<1+k(n)$ and $1<l(n+1)<1+l(n)$. Most delay functions in practice do not satisfy such severe constraints. The delay-dependent stability criterion in our paper removes such constraints. Based on a new Lyapunov functional and techniques based on inequalities, our new delay-dependent stability criterion for discrete-time BAM neural networks with variable delays is given in term of LMIs. Since our result imposes few constraints on the system, it can be applied to a much more general class of BAM neural networks with a broad range of time delay functions.

\section{An illustrative example}

To illustrate how our criterion can be applied to more general delayed BAM neural networks than Theorem 1 in [26], we discuss a BAM neural network with the same coefficients as those in [26] but different delay functions.

Example 1. Consider the following delayed BAM neural network

$$
\begin{aligned}
x_{1}(n+1) & =\frac{1}{5} x_{1}(n)+\frac{1}{8} f\left(y_{2}(n-k(n))\right), \\
x_{2}(n+1) & =\frac{1}{5} x_{2}(n)+\frac{1}{8} f\left(y_{1}(n-k(n))\right), \\
y_{1}(n+1) & =\frac{1}{10} y_{1}(n)-\frac{1}{20} f\left(x_{1}(n-l(n))\right), \\
y_{2}(n+1) & =\frac{1}{10} y_{2}(n)-\frac{1}{20} f\left(x_{2}(n-l(n))\right) .
\end{aligned}
$$

For this example, let $k(n) \equiv 2, l(n)=4+2(-1)^{n}$, and $f(x)=\left(e^{x}-e^{-x}\right) /\left(e^{x}+e^{-x}\right)$. We have

$$
A=\left[\begin{array}{cc}
1 / 5 & 0 \\
0 & 1 / 5
\end{array}\right], \quad B=\left[\begin{array}{cc}
1 / 10 & 0 \\
0 & 1 / 10
\end{array}\right], \quad C=\left[\begin{array}{cc}
0 & 1 / 8 \\
1 / 8 & 0
\end{array}\right], \quad D=\left[\begin{array}{cc}
-1 / 20 & 0 \\
0 & -1 / 20
\end{array}\right]
$$

and $L=M=I_{2}$ where $I_{2}$ is a $2 D$ unit matrix. Obviously, the delay function $l(n)$ in this example does not satisfy the condition: $1<l(n+1)<1+l(n)$ required by Theorem 1 in [26]for example, $l(3)=2$, while $l(4)=6$. Thus, the delay-dependent stability criterion in [26] can not be applied to this simple example. Now, $l_{\max }=6, l_{\min }=2, k_{\max }=2, k_{\min }=2$. By applying Theorem 1 above, if we take $r=2$, we can use the LMI Toolbox [31] to show that the linear matrix inequalities (6) and (7) have solutions: $P=\operatorname{diag}(259.1180,259.1180), Q=$ $\operatorname{diag}(88.9788,88.9788), \Sigma_{1}=\operatorname{diag}(66.0018,66.0018), \Sigma_{2}=\operatorname{diag}(39.2799,39.2799)$. Our Theorem 1 shows that the equilibrium point of this BAM neural network is exponentially stable. 


\section{Conclusion}

This paper has considered the global exponential stability of discrete-time BAM neural networks with variable delays. A delay-dependent exponential stability criterion has been derived by means of a Lyapunov functional and inequality techniques. Unrealistic constraints on the delay functions assumed in [26] have been removed. Our result can be applied to a more general class of BAM neural networks with a broad range of time delay functions. Furthermore, our sufficient condition for global exponential stability is easily checked in practice.

\section{Acknowledgements}

The authors would like to thank the reviewers and editor for their valuable comments and constructive suggestions. This work was supported by the Humanities and Social Science Foundation of the Ministry of Education, P.R. China under Grant No. 06JA790120.

\section{References}

[1] J. Hopfield, Proc. Natl. Acad.Aci. USA 81(1984) 3088.

[2] B. Kosko, Apll. Opt. 26(1987) 4947.

[3] B. Kosko, IEEE. Trans. Systems Man Cybernet. 18 (1988) 49.

[4] H. Jiang, Z. Teng, IEEE. Trans. Circuits Sytems 51(2004) 174.

[5] X. Huang, J. Cao, D. Huang, Chaos, Solitons \& Fractals 24(2005) 885.

[6] C. Feng, R. Plamondon, IEEE Trans. Neural Networks 14(2003) 1560.

[7] Y. Li, Chaos, Solitons \& Fractals 24(2005) 279.

[8] J. H. Park, Chaos, Solitons \& Fractals 29(2006) 446.

[9] X. Liao, K.W. Wong, S.Z. Yang, Phys. Lett. A 316(2003) 55.

[10] S. Arik, V. Tavsanoglu, Neurocomputing, 68(2005) 163.

[11] J. Zhang, Y.Suda, H. Komine, Phys. Lett. A 338(2005) 55.

[12] Y. Li, P. Liu, Math. Comput. Modelling 40(2004) 757.

[13] J. Cao, L. Wang, IEEE. Trans. Neural Networks 13(2002) 457.

[14] X. Liao, J.B. Yu, Int. J. Circuit Theory Appl. 26(1998) 219.

[15] J. Cao, M. Dong, Appl. Math. Comput. 135(2003) 105.

[16] S. Mohamad, Physica D 159(2001) 233.

[17] J. Zhang, Y. Yang. Int. J. Circuit Theory Appl. 29(2001) 185.

[18] S. Mohamad, K. Gopalsamy, Appl. Math. Comput.135(2003) 17.

[19] D. Liu, A.N. Michael, IEEE. Trans. Circuits Sytems 39(1992) 798.

[20] S. Jagannathan, F.L. Lewis, IEEE. Trans. Neural Networks 7(1996) 107.

[21] L. Jin, M.M. Gupta. IEEE. Trans. Neural Networks 7(1996) 1024.

[22] S. Hu, J. Wang, IEEE Trans. Circuits Systems I 49(2002) 1104. 
[23] J. Cao, J. Liang, J. Math. Anal. Appl. 296(2)(2004) 665.

[24] L. Wang, X. Zou, IEEE. Trans. Circuits Sytems II 51(2004) 315.

[25] J. Liang, J. Cao, Chaos, Solitons \& Fractals 22(2004) 773.

[26] J. Liang, J. Cao, D. W.C. Ho, Phys. Lett. A 335(2005) 226.

[27] J. Cao, IEEE. Trans. Circuits Sytems I 48(2001) 494.

[28] J. Cao, Int. J. Systems Sci. 32(2)(2001) 233.

[29] J. Cao, Phys.Lett. A 307(2003) 136.

[30] J. Cao, J. Wang, Neural networks 17(3)(2004) 379.

[31] S. Boyd, L.E.I. Ghaoui, E. Feron, V. Balakrishnan, Linear Matrix Inequalities in System and Control Theory, SIAM, Philadelphia, 1994.

\section{Appendix}

This appendix gives a proof of Theorem 1. The proof is divided into three steps.

\section{Step 1: Construct a discrete Lyapunov function $V(n)$.}

For the real number $r>1$, define the discrete Lyapunov function

$$
\begin{aligned}
V(n) & =V_{1}(n)+V_{2}(n)+V_{3}(n)+V_{4}(n)+V_{5}(n)+V_{6}(n), \quad \text { where } \\
V_{1}(n) & =r^{n} u^{T}(n) P u(n) \\
V_{2}(n) & =\sum_{s=n-k(n)}^{n-1} r^{s} \varphi^{T}(v(s)) \Sigma_{1} \varphi(v(s)) \\
V_{3}(n) & =\sum_{j=-k_{\max }+2}^{-k_{\min }+1} \sum_{s=n+j-1}^{n-1} r^{s} \varphi^{T}(v(s)) \Sigma_{1} \varphi(v(s)) \\
V_{4}(n) & =r^{n} v^{T}(n) Q v(n) \\
V_{5}(n) & =\sum_{s=n-l(n)}^{n-1} r^{s} \psi^{T}(u(s)) \Sigma_{2} \psi(u(s)) \\
V_{6}(n) & =\sum_{i=-l_{\max }+2}^{-l_{\min }+1} \sum_{s=n+i-1}^{n-1} r^{s} \psi^{T}(u(s)) \Sigma_{2} \psi(u(s))
\end{aligned}
$$

here $P, Q$ are positive definite matrices, and $\Sigma_{1}, \Sigma_{2}$ are positive diagonal matrices.

Using Lyapunov's Direct Method, we can investigate the qualitative nature of the solutions of difference equations (4) without actually determining the solutions themselves. In order to analyze the globally exponential stability of the BAM neural network, we calculate differences $\Delta V(n)$ of the Lyapunov function $V(n)$ along the trajectories of the BAM neural network given in Eq. (4). From Eq. (14), we have

$$
\Delta V(n)=\Delta V_{1}(n)+\Delta V_{2}(n)+\Delta V_{3}(n)+\Delta V_{4}(n)+\Delta V_{5}(n)+\Delta V_{6}(n) .
$$


Step 2: Prove $\Delta V(n) \leq 0$.

Since $A$ is a diagonal matrix, $A^{T}=A$. First consider $\Delta V_{1}(n), \Delta V_{2}(n)$, and $\Delta V_{3}$.

$$
\begin{aligned}
\Delta V_{1}(n)= & r^{n+1} u^{T}(n+1) P u(n+1)-r^{n} u^{T}(n) P u(n) \\
= & r^{n+1}\left[u^{T}(n) A P A u(n)+2 u^{T}(n) A P C \varphi(v(n-k(n)))\right. \\
& \left.+\varphi^{T}(v(n-k(n))) C^{T} P C \varphi(v(n-k(n)))\right]-r^{n} u^{T}(n) P u(n) .
\end{aligned}
$$

Noting that $k_{\min } \leq k(n) \leq k_{\max }$, we have

$$
\begin{aligned}
& n-k(n)+1 \leq n+1-k_{\min }, \\
& n+1-k(n+1) \geq n+1-k_{\max } . \\
& \Delta V_{2}(n)=\sum_{s=n+1-k(n+1)}^{n} r^{s} \varphi^{T}(v(s)) \Sigma_{1} \varphi(v(s))-\sum_{s=n-k(n)}^{n-1} r^{s} \varphi^{T}(v(s)) \Sigma_{1} \varphi(v(s)) \\
& =r^{n} \varphi^{T}(v(n)) \Sigma_{1} \varphi(v(n))-r^{n-k(n)} \varphi^{T}(v(n-k(n))) \Sigma_{1} \varphi(v(n-k(n))) \\
& +\sum_{s=n+1-k_{\min }}^{n-1} r^{s} \varphi^{T}(v(s)) \Sigma_{1} \varphi(v(s))+\sum_{s=n+1-k(n+1)}^{n-k_{\min }} r^{s} \varphi^{T}(v(s)) \Sigma_{1} \varphi(v(s)) \\
& -\sum_{s=n-k(n)+1}^{n-1} r^{s} \varphi^{T}(v(s)) \Sigma_{1} \varphi(v(s)) \\
& \leq r^{n} \varphi^{T}(v(n)) \Sigma_{1} \varphi(v(n))-r^{n-k(n)} \varphi^{T}(v(n-k(n))) \Sigma_{1} \varphi(v(n-k(n))) \\
& +\sum_{s=n+1-k_{\min }}^{n-1} r^{s} \varphi^{T}(v(s)) \Sigma_{1} \varphi(v(s))+\sum_{s=n+1-k(n+1)}^{n-k_{\min }} r^{s} \varphi^{T}(v(s)) \Sigma_{1} \varphi(v(s)) \\
& -\sum_{s=n-k_{\min }+1}^{n-1} r^{s} \varphi^{T}(v(s)) \Sigma_{1} \varphi(v(s)) \\
& =r^{n} \varphi^{T}(v(n)) \Sigma_{1} \varphi(v(n))-r^{n-k(n)} \varphi^{T}(v(n-k(n))) \Sigma_{1} \varphi(v(n-k(n))) \\
& +\sum_{s=n+1-k(n+1)}^{n-k_{\min }} r^{s} \varphi^{T}(v(s)) \Sigma_{1} \varphi(v(s)) \\
& \leq r^{n} \varphi^{T}(v(n)) \Sigma_{1} \varphi(v(n))-r^{n-k(n)} \varphi^{T}(v(n-k(n))) \Sigma_{1} \varphi(v(n-k(n))) \\
& +\sum_{s=n+1-k_{\max }}^{n-k_{\min }} r^{s} \varphi^{T}(v(s)) \Sigma_{1} \varphi(v(s)) \text {. } \\
& \Delta V_{3}(n)=\sum_{j=-k_{\max }+2}^{-k_{\min }+1} r^{n} \varphi^{T}(v(n)) \Sigma_{1} \varphi(v(n))- \\
& \sum_{j=-k_{\max }+2}^{-k_{\min }+1} r^{n+j-1} \varphi^{T}(v(n+j-1)) \Sigma_{1} \varphi(v(n+j-1)) \\
& =\left(k_{\max }-k_{\min }\right) r^{n} \varphi^{T}(v(n)) \Sigma_{1} \varphi(v(n))- \\
& \sum_{s=n+1-k_{\max }}^{n-k_{\min }} r^{s} \varphi^{T}(v(s)) \Sigma_{1} \varphi(v(s)) \text {. }
\end{aligned}
$$


Combining Inequality (16) with Eqs. (15) and (17) yields

$$
\begin{aligned}
\Delta V_{1}(n)+\Delta V_{2}(n)+\Delta V_{3}(n) \leq & r^{n+1}\left[u^{T}(n) A P A u(n)+2 u^{T}(n) A P C \varphi(v(n-k(n)))\right. \\
& \left.+\varphi^{T}(v(n-k(n))) C^{T} P C \varphi(v(n-k(n)))\right]-r^{n} u^{T}(n) P u(n) \\
& +\left(k_{\max }-k_{\min }+1\right) r^{n} \varphi^{T}(v(n)) \Sigma_{1} \varphi(v(n)) \\
& -r^{n-k(n)} \varphi^{T}(v(n-k(n))) \Sigma_{1} \varphi(v(n-k(n))) .
\end{aligned}
$$

Since $V_{4}(n), V_{5}(n), V_{6}(n)$ have similar construction to $V_{1}(n), V_{2}(n), V_{3}(n)$ respectively, we can readily show that

$$
\begin{aligned}
\Delta V_{4}(n)+\Delta V_{5}(n)+\Delta V_{6}(n) \leq & r^{n+1}\left[v^{T}(n) B Q B v(n)+2 v^{T}(n) B Q D \psi(u(n-l(n)))\right. \\
& +\psi^{T}(u(n-l(n))) D^{T} Q D \psi(u(n-l(n))]-r^{n} v^{T}(n) Q v(n) \\
& +\left(l_{\max }-l_{\min }+1\right) r^{n} \psi^{T}(u(n)) \Sigma_{2} \psi(u(n)) \\
& -r^{n-l(n)} \psi^{T}(u(n-l(n))) \Sigma_{2} \psi(u(n-l(n))) .
\end{aligned}
$$

Combining Inequality (18) with Inequality (19) implies that

$$
\begin{aligned}
\Delta V(n) \leq & u^{T}(n)\left[r^{n+1} A P A-r^{n} P\right] u(n)+2 r^{n+1} u^{T}(n) A P C \varphi(v(n-k(n))) \\
& +\varphi^{T}(v(n-k(n)))\left[r^{n+1} C^{T} P C-r^{n-k(n)} \Sigma_{1}\right] \varphi(v(n-k(n))) \\
& +\left(k_{\max }-k_{\min }+1\right) r^{n} \varphi^{T}(v(n)) \Sigma_{1} \varphi(v(n)) \\
& +v^{T}(n)\left[r^{n+1} B Q B-r^{n} Q\right] v(n)+2 r^{n+1} v^{T}(n) B Q D \psi(u(n-l(n))) \\
& +\psi^{T}(u(n-l(n)))\left[r^{n+1} D^{T} Q D-r^{n-l(n)} \Sigma_{2}\right] \psi(u(n-l(n))) \\
& +\left(l_{\max }-l_{\min }+1\right) r^{n} \psi^{T}(u(n)) \Sigma_{2} \psi(u(n)) .
\end{aligned}
$$

Since $\Sigma_{1}$ and $\Sigma_{2}$ are diagonal matrices, from Inequality (5), we have

$$
\begin{aligned}
\varphi^{T}(v(n)) \Sigma_{1} \varphi(v(n)) & \leq v^{T}(n) M \Sigma_{1} M v(n) \\
\psi^{T}(u(n)) \Sigma_{2} \psi(u(n)) & \leq u^{T}(n) L \Sigma_{2} L u(n) .
\end{aligned}
$$

Therefore

$$
\begin{aligned}
\Delta V(n) \leq & u^{T}(n)\left[r^{n+1} A P A-r^{n} P\right] u(n)+2 r^{n+1} u^{T}(n) A P C \varphi(v(n-k(n))) \\
& +\varphi^{T}(v(n-k(n)))\left[r^{n+1} C^{T} P C-r^{n-k_{\max }} \Sigma_{1}\right] \varphi(v(n-k(n))) \\
& +\left(k_{\max }-k_{\min }+1\right) r^{n} v^{T}(n) M \Sigma_{1} M v(n) \\
& +v^{T}(n)\left[r^{n+1} B Q B-r^{n} Q\right] v(n)+2 r^{n+1} v^{T}(n) B Q D \psi(u(n-l(n))) \\
& +\psi^{T}(u(n-l(n)))\left[r^{n+1} D^{T} Q D-r^{n-l_{\max }} \Sigma_{2}\right] \psi(u(n-l(n))) \\
& +\left(l_{\max }-l_{\min }+1\right) r^{n} u^{T}(n) L \Sigma_{2} L u(n) \\
= & r^{n} \xi(n)\left[\begin{array}{cc}
r A P A-P+F & r A P C \\
r C^{T} P A & r C^{T} P C-r^{-k_{\max }} \Sigma_{1}
\end{array}\right] \xi(n)^{T}+ \\
& r^{n} \eta(n)\left[\begin{array}{cc}
r B Q B-Q+G & r B Q D \\
r D^{T} Q B & r D^{T} Q D-r^{-l_{\max } \Sigma_{2}}
\end{array}\right] \eta(n)^{T},
\end{aligned}
$$

where $\xi(n)=\left[u^{T}(n), \varphi^{T}(v(n-k(n)))\right]^{T}, \eta(n)=\left[v^{T}(n), \psi^{T}(u(n-l(n)))\right]^{T}$.

From Inequalities (6), (7) and (20), we have

$$
\Delta V(n) \leq 0 .
$$

Hence,

$$
V(n) \leq V(0) .
$$




\section{Step 3: Prove the origin is globally exponentially stable.}

First, we estimate the size of $V(0)$. From Eq. (14) and Inequality (5), we have

$$
\begin{aligned}
V(0) \leq & u^{T}(0) P u(0)+\left(k_{\max }-k_{\min }+1\right) \sum_{s=-k_{\max }}^{-1} r^{s} \varphi^{T}(v(s)) \Sigma_{1} \varphi(v(s)) \\
& +v^{T}(0) Q v(0)+\left(l_{\max }-l_{\min }+1\right) \sum_{s=-l_{\max }}^{-1} r^{s} \psi^{T}(u(s)) \Sigma_{2} \psi(u(s)) \\
\leq & u^{T}(0) P u(0)+\left(k_{\max }-k_{\min }+1\right) \lambda_{\max }\left(\Sigma_{1}\right)\|M\|^{2} \sum_{s=-k_{\max }}^{-1} r^{s} v(s)^{T} v(s) \\
& +v^{T}(0) Q v(0)+\left(l_{\max }-l_{\min }+1\right) \lambda_{\max }\left(\Sigma_{2}\right)\|L\|^{2} \sum_{s=-l_{\max }}^{-1} r^{s} u(s)^{T} u(s) \\
\leq & {\left[\lambda_{\max }(P)+\left(l_{\max }-l_{\min }+1\right) \delta_{1} \lambda_{\max }\left(\Sigma_{2}\right)\|L\|^{2}\right] \sup _{s \in N\left(-l_{\max }, 0\right)}\|u(s)\|^{2} } \\
& +\left[\lambda_{\max }(Q)+\left(k_{\max }-k_{\min }+1\right) \delta_{2} \lambda_{\max }\left(\Sigma_{1}\right)\|M\|^{2}\right] \sup _{s \in N\left(-k_{\max }, 0\right)}\|v(s)\|^{2} \\
\leq & d\left[\sup _{s \in N\left(-l_{\max }, 0\right)}\|u(s)\|^{2}+\sup _{s \in N\left(-k_{\max }, 0\right)}\|v(s)\|^{2}\right] .
\end{aligned}
$$

Next, it follows from Inequalities (21) and (22) that

$$
V(n) \leq d\left[\sup _{s \in N\left(-l_{\max }, 0\right)}\|u(s)\|^{2}+\sup _{s \in N\left(-k_{\max }, 0\right)}\|v(s)\|^{2}\right] .
$$

Again, from Eq. (14), we have

$$
\begin{aligned}
V(n) & \geq V_{1}(n)+V_{4}(n) \\
& =r^{n} u^{T}(n) P u(n)+r^{n} v^{T}(n) Q v(n) .
\end{aligned}
$$

So

$$
V(n) \geq r^{n} \min \left\{\lambda_{\min }(P), \lambda_{\min }(Q)\right\}\left[\|u(n)\|^{2}+\|v(n)\|^{2}\right] .
$$

Combining Inequalities (23) and (25) gives Inequality (8), completing the proof. 